\title{
RECURRENCE OF HYPEROXALURIA AND KIDNEY DISEASE AFTER COMBINED INTESTINE-KIDNEY TRANSPLANTATION FOR ENTERIC HYPEROXALURIA
}

Burcin Ekser, MD, $\mathrm{PhD}^{1}$, Richard S. Mangus, MD, MS ${ }^{1}$, Chandrashekhar A. Kubal, MD, $P h D^{1}$, Jonathan A. Fridell, $M D^{1}$, John A. Powelson, $M D^{1}$, Santosh Nagaraju, MD ${ }^{1}$, Plamen Mihaylov, $\mathrm{MD}^{1}$, Carrie L. Phillips, $\mathrm{MD}^{2}$, Romil Saxena, $\mathrm{MD}^{2}$, William C. Goggins, $\mathrm{MD}^{1}$

(1) Transplant Division, Department of Surgery, Indiana University School of Medicine, Indianapolis, IN, USA; (2) Department of Pathology, Indiana University School of Medicine, Indianapolis, IN, USA.

\section{Short Title: Combined intestine-kidney transplantation}

Address correspondence to:

Burcin Ekser, MD, PhD

Transplant Division, Department of Surgery

Indiana University School of Medicine

550 University Blvd

Room 4601

Indianapolis, IN, 46202

Telephone: 317-948-3835; Fax 317-948-3268

Email: bekser@iupui.edu

Key Words: Enteric hyperoxaluria, Intestinal transplantation, Kidney transplantation Secondary hyperoxaluria

Conflict of Interest: The authors of the present manuscript have no conflict of interest.

(Word counts: Abstract 249; Text 2317; Figures: 4)

This is the author's manuscript of the article published in final edited form as:

Ekser, B., Mangus, R. S., Kubal, C. A., Fridell, J. A., Powelson, J. A., Nagaraju, S., ... Goggins, W. C. (2016).

Recurrence of Hyperoxaluria and Kidney Disease after Combined Intestine-Kidney Transplantation for Enteric Hyperoxaluria. American Journal of Nephrology, 44(2), 85-91. https://doi.org/10.1159/000447785 


\section{ABSTRACT}

Background: Enteric hyperoxaluria $(\mathrm{EH})$ occurs with a rate of $5-24 \%$ in patients with inflammatory bowel disease, ileal resection, and modern bariatric surgery. The excessive absorption of calcium oxalate causes chronic kidney disease (CKD) in patients with $\mathrm{EH}$. In the literature, a single experience was reported in combined intestine-kidney transplantation (CIKTx) in patients with CKD due to $\mathrm{EH}$.

Methods: After a report of two successful cases of CIKTx in patients with EH and CKD, one was performed at our center in a 59-year-old Caucasian female who developed intestinal failure with total parenteral nutrition (TPN)-dependence after a complication post-bariatric surgery. Before CIKTx, she underwent kidney transplantation alone (KTA) twice which failed due to oxalate nephropathy.

Results: In July 2014, the patient underwent CIKTx and bilateral allograft nephrectomy to avoid $\mathrm{EH}$ and oxalate stone burden. The post-operative course was complicated with acute tubular necrosis due to the use of high pressors related to perioperative bleeding. The patient was discharged 79 days after CIKTx with a serum creatinine $(\mathrm{s}-\mathrm{Cr})$ of 1.2 $\mathrm{mg} / \mathrm{dL}$ and TPN-free. Her s-Cr increased at 7 months and a renal biopsy showed oxalate nephropathy. SLC26A6 (oxalate transporter) staining was significantly diminished in native duodenum/rectum as well as in intestinal allograft compared to control.

Conclusions: KTA in patients with CKD secondary to EH should not be recommended due to high risk of recurrence. Although other centers showed good long-term outcomes in CIKTx, our patient experienced recurrence of $\mathrm{EH}$ due to oxalate transporter defect, early kidney allograft dysfunction, and prolonged antibiotic use. 


\section{ABBREVIATIONS}

CIKTx $=$ combined intestine-kidney transplantation

$\mathrm{CKD}=$ chronic kidney disease

eGFR = estimated glomerular filtration rate

ITX = intestinal transplantation

SMA = superior mesenteric artery

SMV = superior mesenteric vein

TPN = total parenteral nutrition 


\section{INTRODUCTION}

A treatment option for intestinal failure in patients with severe complications of total parenteral nutrition (TPN) is intestinal transplantation (ITx) (1). A recent report from the Intestinal Transplant Registry (2,887 various types of ITx from 82 international programs between 1985-2012) showed current actuarial patient survival rates of $76 \%, 56 \%$, and $43 \%$ at 1,5 , and 10 years, respectively (2). In contrast, patients requiring combined intestine/multivisceral and kidney transplantation showed lower patient survival rates than intestine/multivisceral transplants alone $(3,4)$.

Enteric hyperoxaluria (i.e., secondary hyperoxaluria) is a frequent complication of inflammatory bowel diseases, ileal resection, and modern bariatric surgery (5-8). The prevalence of enteric hyperoxaluria has been estimated at $5-24 \%$ of all patients with gastrointestinal diseases associated with malabsorption $(8,9)$. It does not have a genetic predisposition like primary hyperoxaluria, except genetic variations of intestinal oxalate transporters, such as SLC26A6 (9-10). It is primarily due to the excessive absorption of calcium oxalate (9), which causes chronic kidney disease (CKD) requiring renal replacement therapy (8).

Patients with enteric hyperoxaluria requiring kidney transplantation are at risk of the recurrence of disease and calcium oxalate deposition in the renal allograft, which will cause CKD and eventually the loss of the renal allograft. Ceulemans and colleagues (11) reported 2 cases of combined intestine-kidney transplantation (CIKTx) in patients with enteric hyperoxaluria secondary to intestinal failure. In both cases, patients were in 
hemodialysis and TPN-dependent at the time of transplantation and became dialysis and TPN-free after CIKTx at 11 months of follow-up in one case, and at almost 6 years of follow up in another case.

We here report our experience in a patient with enteric hyperoxaluria and CKD who underwent CIKTX. 


\section{METHODS AND RESULTS}

A 59-year-old Caucasian female (KD, weight: 82 kg, length: 167 cm, BMI: 29.4, blood group A+) had a bariatric gastric bypass surgery in 2002 when she was 43 years old. Two years after her bariatric surgery, she underwent a bowel resection due to cecal volvulus and developed intestinal failure and TPN-dependence. At that time, her serum creatinine was $1.2 \mathrm{mg} / \mathrm{dL}$ and estimated glomerular filtration rate (eGFR) was $>60$ $\mathrm{mL} / \mathrm{min} / 1.73 \mathrm{~m}^{2}$. She then gradually developed CKD (5 years after she was diagnosed with intestinal failure). Despite administration of calcium carbonate and restricted oxalate diet, she was diagnosed with CKD stage 5, and started intermittent hemodialysis in March 2009 (Figure 1). A biopsy of the native kidney showed severe oxalate nephropathy and $24 \mathrm{~h}$ urine oxalate measured by Litholink (Chicago, IL) resulted $60 \mathrm{mg} /$ day.

At this point, the diagnosis of enteric hyperoxaluria was made. She received her first kidney transplantation in July 2009 from a living donor. She underwent bilateral native nephrectomy due to oxalate stone burden 15-months after her kidney transplantation. Unfortunately, hyperoxaluria recurred and she developed CKD again, 15-months after the kidney transplantation. A biopsy of the renal allograft showed severe oxalate nephropathy and $24 \mathrm{~h}$ urine oxalate was $60 \mathrm{mg} /$ day. She went back on hemodialysis in May 2011. Five months after being on hemodialysis, another living donor became available to her and she underwent her $2^{\text {nd }}$ kidney transplantation in October 2011 when she was 52 years old (Figure 1). Less than a year after her $2^{\text {nd }}$ kidney transplantation, 
she developed CKD and the allograft biopsy showed severe oxalate nephropathy in September 2012 (Figure 2A). About 2 years after her $2^{\text {nd }}$ kidney transplantation, she was diagnosed with CKD stage 5 and she restarted on hemodialysis.

In July 2014, when she was 59 years old, a 9-year-old female deceased donor became available. Both T and B cell cross-matches were negative and both class I and II panel reactive antibodies were 0 . She underwent combined intestine-kidney $\left(3^{\text {rd }}\right)$ transplantation to avoid enteric hyperoxaluria related to her intestinal failure. At the same time, she also underwent bilateral allograft nephrectomy (2 previous kidney transplants) to avoid oxalate stone burden. The intestine (which included the entire jejunum, entire ileum, ileocecal valve, cecum, ascending colon, and transverse colon) was transplanted by anastomosing the donor superior mesenteric artery (SMA) to the recipient SMA and the donor superior mesenteric vein (SMV) to the recipient SMV. Proximally, the bowel anastomosis was performed in side-to-side fashion between donor and recipient jejunum. Distally, the donor transverse colon was anastomosed to the recipient sigmoid colon in end-to-side fashion without creating any stoma. After the transplantation of the intestine, the kidney was transplanted in the left iliac fossa intraperitoneally anastomosing renal artery to the external iliac artery and renal vein to the external iliac vein in end-to-side fashion. The ureter was anastomosed using LichGregoir technique with double J stent. The immunosuppressive regimen was consisted of induction of antithymocyte globulin ( $2 \mathrm{mg} / \mathrm{kg} \times 3$ doses), rituximab $\left(150 \mathrm{mg} / \mathrm{m}^{2} \times 1\right.$ dose), and methylprednisolone (3 boluses only with antithymocyte globulin induction). The maintenance immunosuppression included tacrolimus (12h trough levels of 10 
$\mathrm{ng} / \mathrm{mL}$ in the first 3 months and $8 \mathrm{ng} / \mathrm{mL}$ thereafter) and mycophenolate mofetil (1000 $\mathrm{mg} \times 2 /$ daily). Due to the development of leukopenia and persistent diarrhea mycophenolate mofetil was discontinued at 2 months and azathioprine $(100 \mathrm{mg} /$ daily $)$ was added once leukopenia resolved.

The post-operative course was complicated with acute tubular necrosis and delayed graft function of the kidney allograft due to (i) the use of high pressors related to intraoperative bleeding and (ii) hemorrhage due to the accidental removal of a femoral arterial line on post-operative day 1 . The patient had a significant left groin hematoma requiring several units of blood and blood products transfusion. She was placed on a continuous veno-venous hemodialysis for 7 days. On post-operative day 15 , she developed a urinary tract infection for which she was treated with cefepime for 10 days. She was also treated for several blood stream and urinary tract infection episodes with daptomycin, linezolid, meropenem, and micafungin, and their combinations until discharge. On post-operative day 25 , she developed an acute kidney injury and renal dysfunction due to high levels of tacrolimus for which she was supported with a continuous veno-venous hemodialysis for another 12 days.

The patient experienced prolonged diarrhea despite the use of anti-motility agents and couldn't gain weight. Stool fat and stool pancreas elastase were measured. Although stool fat was normal, pancreas elastase was low in the stool $(<50$ microElastase/gm stool), indicating exocrine pancreas insufficiency. The patient started pancrealipase orally and she experienced more formed stools, gained weight with improved 
prealbumin levels. Despite exocrine pancreas insufficiency, her endocrine pancreas function was normalized 15 days after the transplant (high blood glucose in the first 15 days probably due to steroid induction). The patient was discharged on post-operative day 79 dialysis-free with a serum creatinine of $1.2 \mathrm{mg} / \mathrm{dL}$ and TPN-free.

She developed a slow deterioration of her renal function over the next 3 months (6 months post-transplant). At 7 months after the $3^{\text {rd }}$ kidney transplantation (March 2015), her serum creatinine was $3.3 \mathrm{mg} / \mathrm{dL}$ (Figure 3). A biopsy of the renal allograft showed intraluminal oxalate crystals, oxalate nephropathy, and Banff la acute cellular rejection (Figure 2B), which was treated with steroid boluses and IV fluids. Her 24h urine oxalate level was $38 \mathrm{mg} /$ day (Figure 1). Although she is currently TPN-free, she has started on hemodialysis on March 2016 (20 months after CIKTx). 


\section{DISCUSSION}

Enteric hyperoxaluria and related renal dysfunction was first described in early 70 ies as acquired hyperoxaluria in patients (both adults and children) with resection of the distal ileum $(5,6)$. Several medical strategies have been applied to reduce oxalate absorption, such as reduced oxalate or oxalate-free diets, administration of oral calcium and magnesium salts, and more recently the use of oxalate degrading bacteria (Oxalobacter formigens) containing probiotics (9).

The problem of enteric hyperoxaluria progressing to CKD became more evident despite abovementioned medical therapies since early 2000 with an increase in modern bariatric surgeries (12). Kidney transplantation alone was reported in patients with CKD secondary to enteric hyperoxaluria with $(13,14)$ or without $(15,16)$ success.

Despite several CIKTxs were reported by the Intestinal Transplant Registry, the first and only report on CIKTx in patients with enteric hyperoxaluria and CKD was published in 2013 by Ceulemans and colleagues (11). They reported 2 successful cases of the resolution of intestinal failure and CKD secondary to enteric hyperoxaluria following CIKTx. After Ceulemans et al.'s success, we reevaluated our patient after 2 failed kidney transplantations due to the recurrence of enteric hyperoxaluria.

In our case, the genetic testing was performed to exclude primary hyperoxaluria between the first and second kidney transplantation. All 3 subtypes of primary hyperoxaluria were tested for AGXT, GRHPR, and DHDPSL gene mutations, which 
resulted negative. Negative results were interpreted as the patient having $<0.5 \%$ of chances of genetic defect in her oxalate metabolism. There was no bone or heart involvement due to hyperoxaluria. Her liver enzymes were also completely normal. It was then decided to proceed with her second kidney transplantation. After the failure of $2^{\text {nd }}$ kidney transplantation, we repeated all genetic testing for primary hyperoxaluria and performed a liver biopsy to decide whether we should have proceeded with combined multivisceral (which includes the liver) and kidney transplantation or isolated intestine and kidney $\left(3^{\text {rd }}\right)$ transplantation. Genetic testing for all subtypes of primary hyperoxaluria resulted negative again. The liver biopsy showed normal liver architecture and hepatocytes with no fibrosis (not shown). Clinically, all liver function tests were normal. It was then decided to proceed with CIKTx instead of multivisceral transplant and kidney transplantation.

Post-operatively, our patient experienced the delayed graft function of the renal allograft due to perioperative complications. She was on warfarin therapy before transplant due to deep venous thrombus in her right leg. Despite careful hemodynamics and correction of her high INR, she required increased volume of blood and blood product transfusions during the transplant surgery, which also included two allograft nephrectomies. The hemorrhage after an accidental removal of a femoral arterial line was difficult to control. She developed a big hematoma in her leg and thigh and required several units of blood transfusion, which added another burden on the function of the renal allograft.

In order to exclude another genetic defect in oxalate transporter (SLC26A6) as shown 
by Capolongo et al (10), biopsies from patient's native duodenum, native rectum, and intestine allograft ( $\mathrm{x} 2-4$ and 10 months after $\mathrm{CIKTx})$ were stained (Figure 4). Although the normal intestinal mucosa (from the $3^{\text {rd }}$ party, served as control) showed intense staining of the subnuclear as well as supraneuclear cytoplasm of the epithelial cells, biopsies of native duodenum and rectum from our patient, and the small intestinal allograft showed a significant less intensity of staining. Furthermore, staining was restricted to the subnuclear portion of the cytoplasm whereas the supranuclear cytoplasm was negative. Some cells did not stain at all or stained very weakly (Figure 4).

There were several reasons behind the recurrence of hyperoxaluria and oxalate nephropathy post-CIKTx. (i) Although we found the weak expression of oxalate transporter (SLC26A6) both in native intestine and intestinal allograft after the recurrence, we believe that this phenomenon could be the strongest reason for poor oxalate elimination (9-10). Moreover, other contributing reasons would be (ii) the kidney allograft with early dysfunction which was not able to excrete high levels of oxalate and (iii) the prolonged (>2 months) consumption of antibiotics post-transplantation interfering with the intestinal flora and leading to enteric hyperoxaluria, as shown by Lefaucheur et al (17) in native kidneys following nonrenal solid organ transplantation, such as heart, lung, and liver transplantation. We believe that mycophenolate mofetil-induced enteric hyperoxaluria, as shown by Jahromi et al (18) is not the case in our patient since mycophenolate mofetil was discontinued at 2 months post-transplantation due to its presumed side effects, such as diarrhea and bone marrow suppression. 
The genetic defect in oxalate transporter (SLC26A6) in native duodenum and rectum cannot be corrected. However, if it was known before CIKTx, the strategy of performing a full multivisceral transplant (with the liver and duodenum) could have been considered. There is also an unknown question; whether the defect in oxalate transporter in the remaining portion of native duodenum $(15-20 \mathrm{~cm})$ and rectum $(15-$ $20 \mathrm{~cm}$ ) would suffice to induce oxalosis over full length working transplanted intestine. Moreover, in our case, the expression of SLC26A6 was significantly less intense in the transplanted small intestine as well, which adds another unknown in the field whether this was isolated to our case or all small intestinal transplants would have diminished SLC26A6 expression.

The early dysfunction of the renal allograft due to the use of high pressors and blood transfusion could be avoided by the delayed implantation of the kidney allograft in CIKTx, as shown in combined liver-kidney transplantation by our group (19). We usually delay the implantation of the kidney allograft up to 3 days in combined liverkidney transplantation, supporting the kidney allograft by continuous hypothermic machine perfusion (19). The delayed implantation of kidney allograft in combined liverkidney transplantation enabled us to hemodynamically stabilize our recipients post-liver transplantation, which offers a less hostile environment for the kidney allograft. The delayed approach kidney implantation allowed us to overcome the burden of the delayed graft function ( $0 \%$ in combined liver and delayed kidney transplantation). 
Alternative strategy to reduce the stress and oxalate burden could be performing bilateral allograft nephrectomies prior to CIKTx. However, in order to maintain minimal urine output and lack of experience with CIKTx in patients with CKD and enteric hyperoxaluria at our center as well as in the literature, we performed bilateral allograft nephrectomies at the time of CIKTx.

In conclusion, we believe that kidney transplantation alone in patients with CKD secondary to enteric hyperoxaluria should not be recommended due to high risk of recurrence. Although other centers showed good long-term outcomes in CIKTX, our patient experienced the recurrence of hyperoxaluria and chronic kidney disease due to the combination of the genetic defect in oxalate transporter, early kidney allograft dysfunction, and the prolonged consumption of antibiotics post-transplantation. Although the defect in the expression of oxalate transporter would be quite difficult to exclude in intestinal or kidney allografts before transplantation, the consideration should be given to avoid early kidney allograft dysfunction and the preservation of the intestinal flora. 


\section{ACKNOWLEDGEMENTS}

No funding was provided for the present study. 


\section{REFERENCES}

1. Fishbein TM. Intestinal transplantation. N Engl J Med 2009; 361: 998-1008.

2. Grant $D, A b u-E l m a g d ~ K$, Mazariegos G, et al. Intestinal transplant registry report: global activity and trends. Am J Transplant 2015; 15: 210-219.

3. Abu-Elmagd KM, Kosmach-Park B, Costa G, et al. Long-term survival, nutritional autonomy, and quality after life after intestinal and multivisceral transplantation. Ann Surg 2012; 256: 494-508.

4. Mangus RS, Tector AJ, Kubal CA, Fridell JA, Vianna RM. Multivisceral transplantation: expanding indications and improving outcomes. J Gastrointest Surg 2013; 17: 179-186.

5. Smith LH, Fromm H, Hofmann AF. Acquired hyperoxaluria, nephrolithiasis, and intestinal disease. N Engl J Med 1972; 286: 1371-1375.

6. Valman HB, Oberholzer VG, Palmer T. Hyperoxaluria after resection of ileum in childhood. Arch Dis Child 1974; 49: 171-173.

7. Whitson JM, Stackhouse GB, Stoller ML. Hyperoxaluria after modern bariatric surgery: case series and literature review. Int Urol Nephrol 2010; 42: 369-374.

8. Nazzal L, Puri S, Goldfarb DS. Enteric hyperoxaluria: an important cause of endstage kidney disease. Nephrol Dial Transplant 2015; ahead of pub. Doi: 10.1093/ndt/gfv005.

9. Robjin S, Hoppe B, Vervaet BA, D'Haese PC, Verhulst A. Hyperoxaluria: a gut-kidney axis? Kidney Int 2011; 80: 1146-1158. 
10. Capolongo G, Abul-Ezz S, Moe OW, Sakhaee K. Subclinical celiac disease and crystal-induced kidney disease following kidney transplant. Am J Kid Dis 2012; 60: 662667

11. Ceulemans LJ, Nijs Y, Nuytens F, et al. Combined kidney and intestinal transplantation in patients with enteric hyperoxaluria secondary to short bowel syndrome. Am J Transplant 2013; 13: 1910-1914.

12. Hassan I, Juncos LA, Milliner DS, Sarmiento JM, Sarr MG. Chronic renal failure secondary to oxalate nephropathy: a preventable complication after jejunoileal bypass. Mayo Clin Proc 2001; 76: 758-760.

13. Rifkin SI. Transplantation for renal failure secondary to enteric hyperoxaluria: a case report. J Med Case Rep 2007; 1: 31.

14. Roberts RA, Sketris IS, MacDonald AS, Belitsky P. Renal transplantation in secondary oxalosis. Transplantation 1988; 45: 985-986.

15. Lim PB, Klebe B, Kon SP. Nephrocalcinosis in a patient with secondary hyperoxaluria. Nephrol Dial Transplant 2004; 19: 1325.

16. Cuvelier C, Goffin E, Cosyns JP, Wauthier M, van Ypersele de Strihou C. Enteric hyperoxaluria: a hidden cause of early renal graft failure in two successive transplants: spontaneous late graft recovery. Am J Kidney Dis 2002; 40 (E3): 1-6.

17. Lefaucheur C, Hill GS, Amrein C, et al. Acute oxalate nephropathy: a new etiology for acute renal failure following nonrenal solid organ transplantation. Am J Transplant 2006; 6: 2516-2521.

18. Jahromi H, Roberts IS, Winearls CG, Vaidya A. Acute renal failure secondary to oxalosis in a recipient of a simultaneous kidney-pancreas transplant: was 
mycophenolate the cause? Nephrol Dial Transplant 2008; 23: 2409-2411

19. Ekser B, Mangus RS, Fridell JA et al. A novel approach in combined liver-kidney transplantation with long-term outcomes. Ann Surg 2016; doi:

10.1097/SLA.0000000000001752. 
Figure Legends:

Figure 1: Time-line of surgeries and major events.

(\#) native kidney biopsy = severe oxalate nephropathy

$\left({ }^{*}\right)$ biopsy of the $1^{\text {st }}$ kidney transplant $=$ severe oxalate nephropathy

$\left.{ }^{* *}\right)$ biopsy of the $2^{\text {nd }}$ kidney transplant $=$ severe oxalate nephropathy

$\left(^{* * *}\right)$ biopsy of the $3^{\text {rd }}$ kidney transplant $=$ severe oxalate nephropathy

( ) 02/2014= work-up for possible multivisceral / isolated intestinal + kidney

transplantation. Liver biopsy showed normal liver architecture, and no fibrosis. Blood

liver function tests were normal. Genetic testing for primary hyperoxaluria (AGXT /

GRHPR / DHDSPL mutations) was negative. Therefore, combined isolated intestinal + kidney transplantation was decided to perform.

$\mathrm{CKD}=$ chronic kidney disease, eGFR= estimated glomerular filtration rate, $\mathrm{HD}=$ hemodialysis, IF = intestinal failure, TPN = total parenteral nutrition

Figure 2: Biopsy of kidney allografts

(A) Biopsy of the second kidney allograft: H\&E (x40) of the second kidney allograft showing oxalate crystal.

(B) Biopsy of the third kidney allograft: H\&E (x40) of the third kidney allograft showing oxalate crystals in non-polarized and polarized light microscopy.

Figure 3: Serum creatinine (S-Cre) levels over the years.

Serum creatinine levels from July 2009 to December 2015.

Figure 4: Oxalate transporter (SLC26A6) staining.

(A) Control normal small intestinal mucosa: Intense staining of the subnuclear as well as supranuclear cytoplasm of the epithelial cells.

(B) Native duodenum: Less intensity of staining only in the subnuclear cytoplasm. 
(C) Native rectum: almost no staining.

(D) Small intestine allograft (4 months after the transplant): less intensity staining compared to control only in the subnuclear cytoplasm of the epithelial cells.

(E) Small intestine allograft (10 months after the transplant): less intensity staining compared to control only in the subnuclear cytoplasm of the epithelial cells but better than 4 months after the transplant.

Three micron thick sections were stained immunohistochemically with an antibody against SLC26A6 (Novus Biologicals, Littleton, CO). Briefly, sections from formalin fixed paraffin embedded tissues were deparaffinized and cleared in xylene. Antigen retrieval was carried out with EDTA at pH8. After blocking endogenous peroxidase, the sections were incubated with the primary antibody at a dilution of 1:1000 for 20 minutes at room temperature. The reaction was visualized using the Dako Flex Plus kit (Dako, Carpinteria, CA) using 3-3'-diaminobenizidine tetra hydrochloride as the chromogen. All images have $\times 1000$ magnification. 
Figure 1:

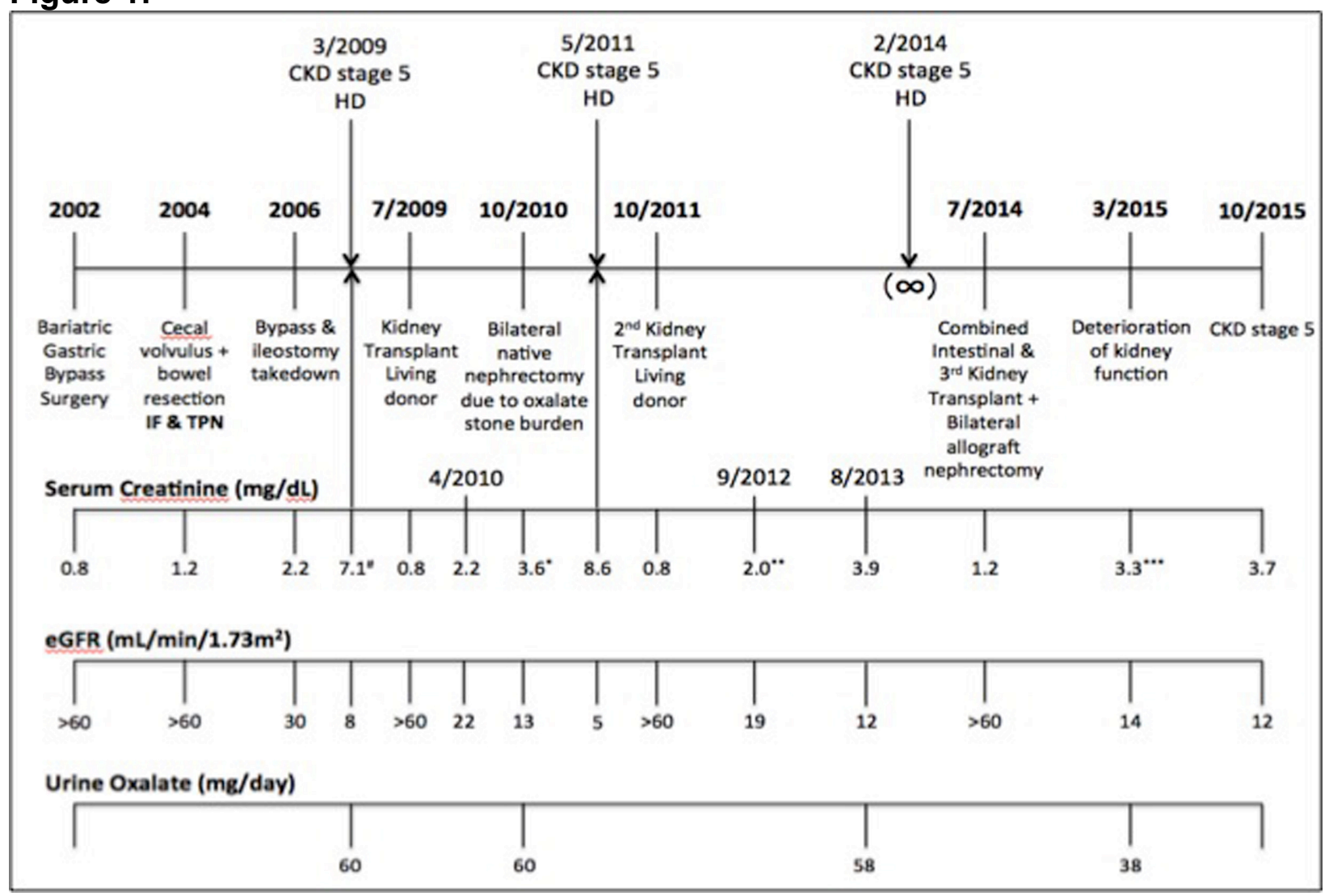


Figure 2A:

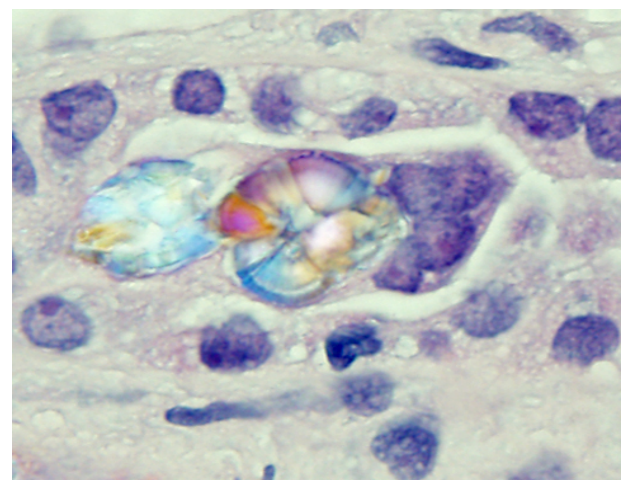

Figure 2B:

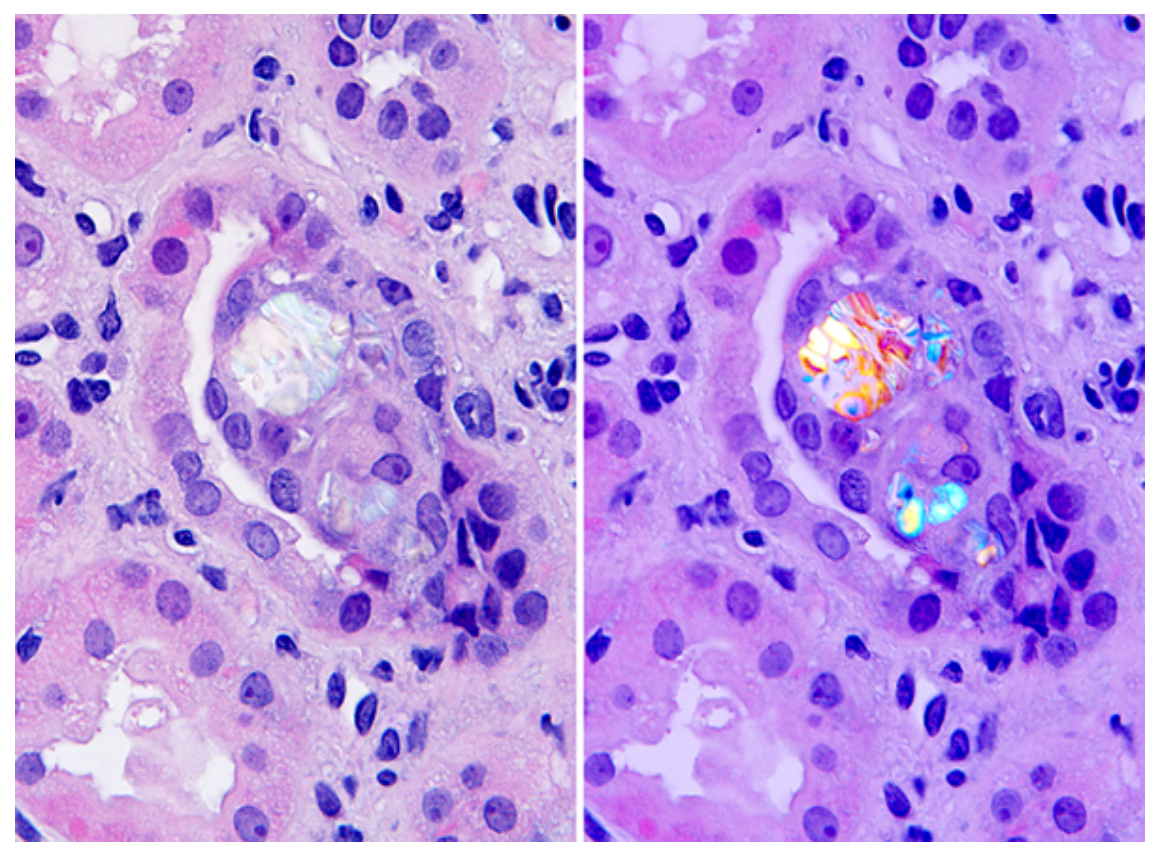


Figure 3:

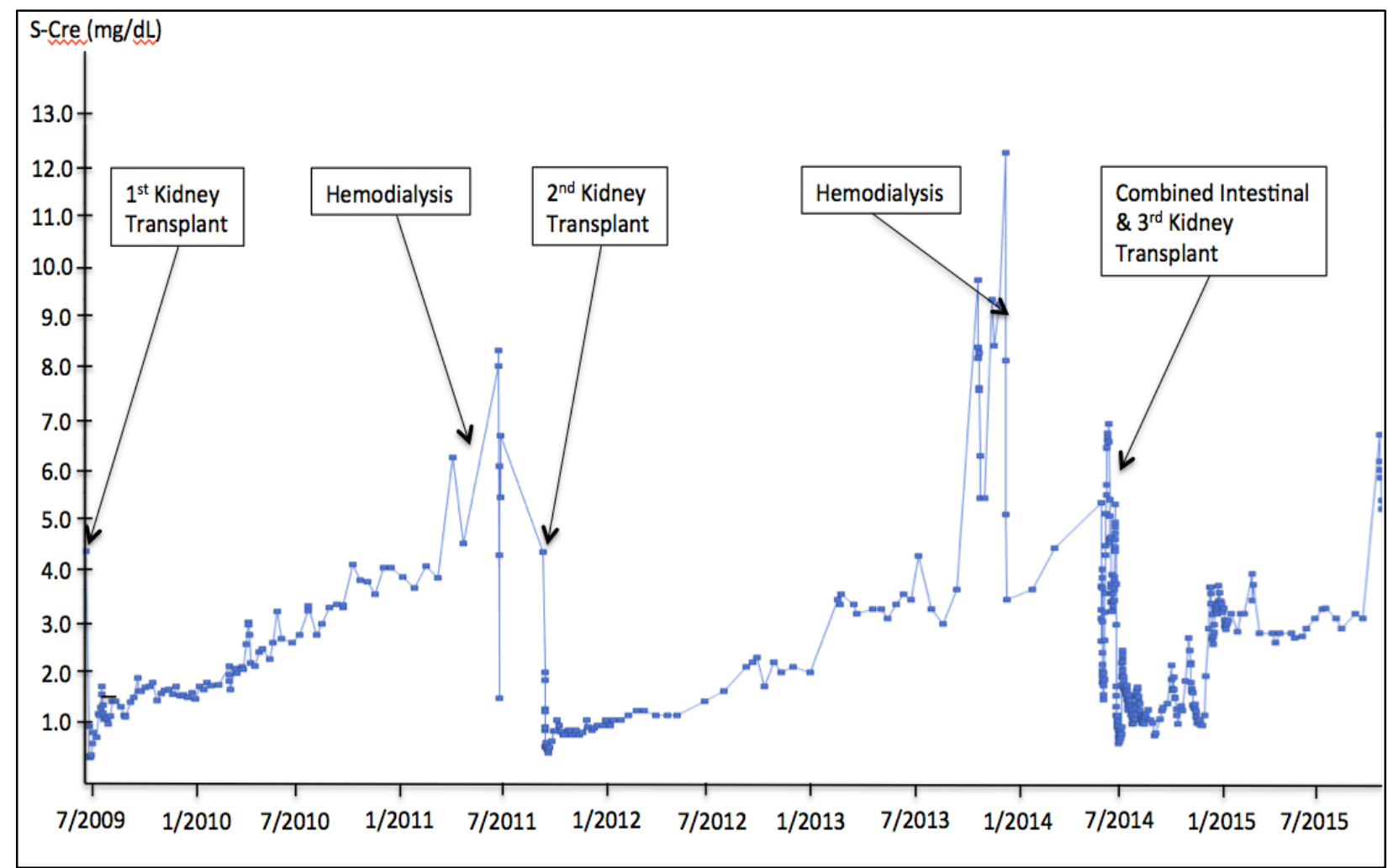


Figure 4:

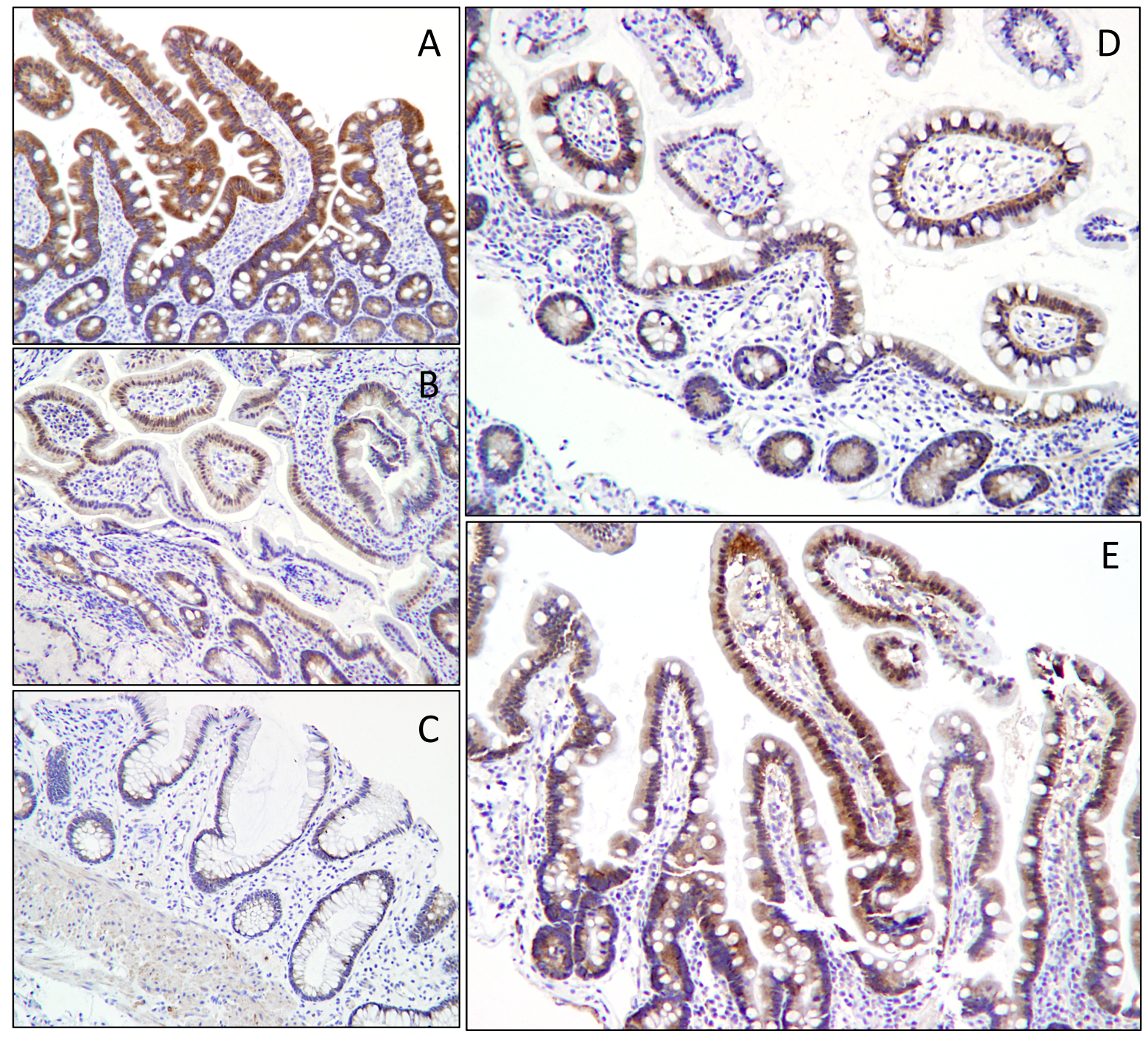

